\title{
Differential expression and function of the caveolin-1 gene in non-small cell lung carcinoma
}

\author{
HONG LEI CHEN, LI FANG FAN, JUN GAO, JING PING OUYANG and YU XIA ZHANG \\ Department of Pathology, School of Basic Medicine Science, Wuhan University, Wuhan 430071, P.R. China
}

Received July 16, 2010; Accepted September 21, 2010

DOI: $10.3892 /$ or.2010.1095

\begin{abstract}
This study was designed to clarify the function of caveolin-1 (Cav-1) in the development of lung cancer by investigating the mutation and protein expression of the Cav-1 gene in non-small cell lung carcinoma (NSCLC). Quantum dot immunofluorescence histochemistry was used to evaluate Cav-1 protein expression and subcellular localization in the lung cancer tissue microarray including 140 cases of lung cancer and 20 cases of non-cancerous lung tissue. Mutation of the Cav-1 gene in exon 1 and exon 3 was detected by polymerase chain reaction-single strand conformation polymorphism and sequencing. The positive rates of Cav-1 expression were 49.3\% (69/140) in NSCLC group, significantly lower than the $100 \%(20 / 20)$ rate in the control group. Adenocarcinomas (16.7\%), adenosquamous carcinomas (38.4\%), squamous cell carcinomas $(67.1 \%)$ and large cell lung cancers $(66.7 \%)$ displayed Cav-1 positive staining, suggesting a gradient of Cav-1 expression according to tumor histotype-related aggressiveness. High-expression of Cav-1 protein was statistically correlated with pathologic TNM stage and lymph node metastasis. No mutation could be detected in exon 1 and exon 3 from all Cav-1 protein negative expression of NSCLC samples. Cav-1 immunoreactivity in lung cancer is histotype-dependent, increased Cav-1 expression indicates the malignant progression and high invasion features of NSCLCs. Deregulation of Cav-1 expression in NSCLCs may not correlate with mutation.
\end{abstract}

\section{Introduction}

Lung cancer is one of the most common malignant tumors in humans with high incidence and mortality rate. In China, there were about 0.50 million lung cancer cases and 0.43 million deaths in 2005 (1). About $80-85 \%$ of lung cancers are non-small cell lung cancer (NSCLC), of which adenocarcinoma (AC) and squamous cell carcinoma (SCC) are the

Correspondence to: Dr Hong Lei Chen or Dr Yu Xia Zhang, Department of Pathology, School of Basic Medicine Science, Wuhan University, Donghu Road 115, Wuhan 430071, P.R. China

E-mail: chenhlwhu@gmail.com

E-mail: zhangyuxiacn@hotmail.com

Key words: caveolin-1, lung carcinoma, mutation, quantum dots two major types. The etiology and mechanism of lung carcinogenesis are still unclear. The development of tumor correlates with the pathway aberration of signal transduction.

Caveolin is the major structural protein in caveolae. To date, multiple forms of caveolin, caveolin-1, -2 and -3 , have been identified. Caveolin-1 (Cav-1) functionally regulates the activity of many signaling molecules, such as the Wnt/Bcatenin/lef-1 (2), Rac1 (3) and Mdm2/p53-mediated pathway (4), which are potentially involved in the development of human cancer. The role of Cav-1 in cancer cells is still debated and highly controversial. Cav-1 is down-regulated, e.g., in human ovarian carcinoma (5), colon AC (6) and head and neck SCC (7), this function strongly suggests the possible role of Cav-1 as a tumor suppressor. Consistent with this, the human caveolin gene is mapped to a suspected tumor locus (7q31.1), which is often deleted in human cancers (8). Conversely, Cav-1 overexpression is associated with more aggressive behaviour, increased recurrence rate and poorer prognosis in prostatic cancer (9), pulmonary SCC (10) and hepatocellular carcinoma (11). Due to such discrepancies, it is still unclear whether Cav-1 up- or down-regulation is responsible for a biological advantage in tumorigenesis.

Advances in nanotechnology have led to the development of novel fluorescent probes called quantum dots (QDs). QDs are novel nanocrystal fluorophores with extremely high fluorescence efficiency and minimal photobleaching. They also possess a constant excitation wavelength together with sharp and symmetrical tunable emission spectra. These unique optical properties make them near-perfect fluorescent markers and there has recently been rapid development of their use for bioimaging (12). Our previous studies have shown the way for the general application of QDs to immunohistochemistry (IHC) in formalin-fixed and paraffin-embedded tissue and subsequently been used to detect a vast array of proteins (13).

In the present study, we examined the expression patterns of Cav-1 in different histology of human lung cancer. We analyzed 140 NSCLC and 20 non-cancerous lung tissues by QDs immunofluorescence histochemical staining on a tissue microarray for possible relationships between the Cav-1 expression and clinicopathological parameters and screened for mutations in the Cav-1 gene.

\section{Materials and methods}

Patients and samples. A total of 140 NSCLCs and 20 noncancerous lung tissues (used as controls) were included in 
Table I. Relationship between expression of Cav-1 protein and clinicopathological parameters of the NSCLCs.

\begin{tabular}{|c|c|c|c|c|c|c|}
\hline \multirow[b]{2}{*}{ Group } & \multirow[b]{2}{*}{ Cases } & \multicolumn{3}{|c|}{ Caveolin-1 protein } & \multirow[b]{2}{*}{$\chi^{2}$ value } & \multirow[b]{2}{*}{ P-value } \\
\hline & & Negative & Positive & Positive rate $(\%)$ & & \\
\hline Age & & & & & 1.059 & 0.303 \\
\hline$<57$ & 65 & 36 & 29 & 44.6 & & \\
\hline$\geq 57$ & 75 & 35 & 40 & 53.3 & & \\
\hline Gender & & & & & 0.125 & 0.723 \\
\hline Male & 114 & 57 & 57 & 50.0 & & \\
\hline Female & 26 & 14 & 12 & 46.2 & & \\
\hline \multicolumn{7}{|l|}{ Histology } \\
\hline $\mathrm{SCC}^{\mathrm{a}}$ & 82 & 27 & 55 & 67.1 & 28.228 & $0.000^{\mathrm{a}, \mathrm{b}}$ \\
\hline $\mathrm{AC}^{\mathrm{b}}$ & 42 & 35 & 7 & 16.7 & 3.948 & $0.064^{\mathrm{a}, \mathrm{c}, \mathrm{e}}$ \\
\hline $\mathrm{ASC}^{\mathrm{c}}$ & 13 & 8 & 5 & 38.4 & 4.375 & $0.097^{\mathrm{b}, \mathrm{d}, \mathrm{e}}$ \\
\hline $\mathrm{LCLC}^{\mathrm{d}}$ & 3 & 1 & 2 & 66.7 & & \\
\hline TNM stage & & & & & 4.016 & 0.045 \\
\hline $\mathrm{I}$ & 102 & 57 & 45 & 44.1 & & \\
\hline II+III & 38 & 14 & 24 & 63.2 & & \\
\hline $\mathrm{N}$ classification & & & & & 5.857 & 0.016 \\
\hline $\mathrm{N}_{0}$ & 104 & 59 & 45 & 43.3 & & \\
\hline $\mathrm{N}_{1-3}$ & 36 & 12 & 24 & 66.7 & & \\
\hline
\end{tabular}

SCC, squamous cell carcinoma; AC, adenocarcinoma; ASC, adenosquamous carcinoma; LCLC, large cell lung cancer. ${ }^{\mathrm{e} T h e}$ Fisher's exact test.

this study, obtained from patients of Zhongnan Hospital of Wuhan University. Clinical data were reviewed to ensure the clinical stage and metastasis. For each case, the haematoxylin and eosin sections were reviewed by at least two pathologists following the 2004 World Health Organization histological classification for lung cancers. The clinicopathological parameters are summarized in Table I. Of the tumor samples 82 were classified as SCCs, 42 as ACs including 8 bronchiolo-alveolar carcinoma and 7 papillary adenocarcinoma, 13 as adenosquamous carcinomas (ASCs), and 3 as large cell lung cancers (LCLCs). This study was approved by the Ethics Committee of Wuhan University.

Tissue microarray construction. Tissue microarray blocks were constructed using a tissue-arraying instrument (Beecher Instruments, MD, USA) (14). Two replicates of tumor tissue $1.1 \mathrm{~mm}$ in diameter from at least two different paraffin wax blocks from each case were placed in tissue microarray blocks from 140 NSCLC patients. Non-cancerous lung tissues were obtained from 20 patients and two replicates were included in the same tissue microarrays. Each slide has $>95 \%$ tissue core retention.

Quantum dot immunofluorescence histochemical staining. Cav-1 immunoreactivity was tested in all tissue microarrays using anti-Cav-1 antibodies (diluted 1:300; Santa Cruz, USA) and performed using QDs immunofluorescence histochemistry
(QDs-IHC) according to the manufacturer's instructions (Wuhan Jiayuan Quantum Dot Co. Ltd.). Antigen retrieval was performed using citric acid $(10 \mathrm{mM}, \mathrm{pH} 6.0)$ at $95^{\circ} \mathrm{C}$ for $10 \mathrm{~min}$ and left to cool for $30 \mathrm{~min}$. The detailed procedure was performed as previously described (13). The signal obtained from the labeling of cells was detected by Olympus BX51 fluorescence microscopy (CCD DP72). The immunofluorescence signals were colored red (605 nm QDs), bright, and photo stable. Positive staining of Cav-1 was located in the cell membrane and cytoplasm. Negative controls were carried out by omitting the primary antibody instead of TBS. Normal blood vessel endothelial cells within the tumor section, naturally abundant in Cav-1, were used as positive internal controls (Fig. 1).

Scoring analyses of immunofluorescence results. At least 100 cells were randomly selected and counted from five representative fields of each core using a $20 \mathrm{X}$ objective in a blinded manner by two independent observers (L.F.F. and Y.X.Z.). Positive rates of Cav-1 detected by QDs-IHC staining were assessed according to Kato et al (15). In ACs, we classified them into two groups as (-) and (+) by the threshold between $0 \%$ and the others because most tumors had Cav-1-positive cells $<5 \%$. In SCCs, ASCs and LCLCs, we defined the cut-off between 0-25 and $25-100 \%$ because $25 \%$ level was the most significant pathologic effect in clinicopathological parameters (Table I). 

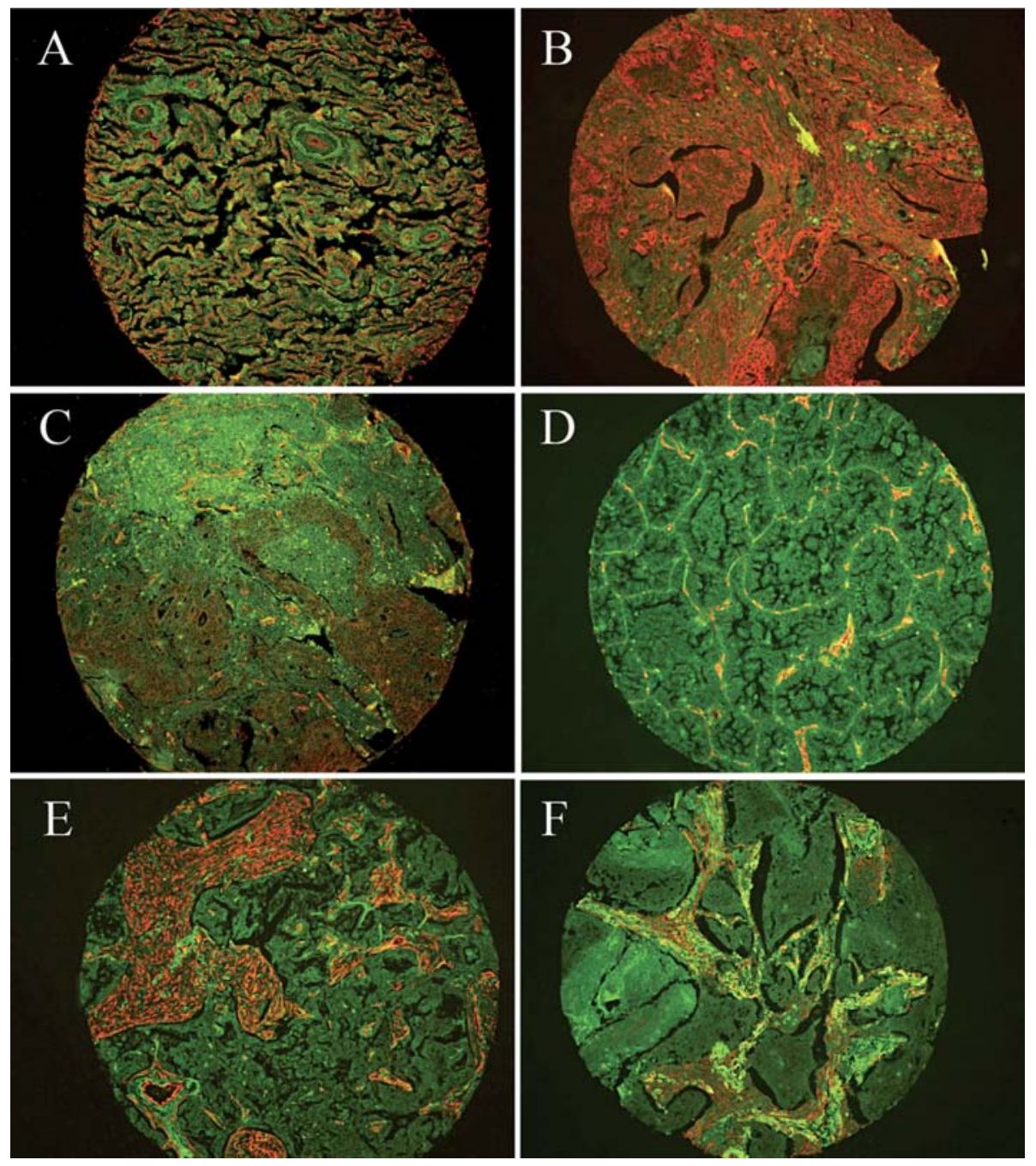

Figure 1. Quantum dot immunofluorescence histochemical staining for Cav-1 in the lung cancer TMAs. (A) Strong staining of Cav-1 in the capillary endothelium cells, type 1 pneumocytes, fibroblasts, smooth muscle cells. (B) Strong staining of Cav-1 in SCC tumor cells and stroma. (C) Positive staining of Cav-1 in AC tumor cells. (D) Negative staining of Cav-1 in bronchioloalveolar carcinomas, blood vessel endothelial cells serve as a positive control. (E) Negative staining of Cav-1 in AC tumor cells but strong staining in the stroma. (F) Week staining of Cav-1 in SCC tumor cells but strong staining in the stroma. Magnification x100.

Cav-1 mutation screening in lung carcinomas. Thirty tumor samples with Cav-1 negative expression and 12 non-cancerous lung tissues were screened for mutations in exons 1 and 3 of the Cav-1 gene. DNA was extracted using the QIAamp DNA Mini Kit (Qiagen) and eluted with $100 \mu 1 \mathrm{H}_{2} \mathrm{O}$. A 159-bp fragment was amplified from exon 1 (GenBank NM AF095591, F: 5'-AGTTCCCTTAAAGCACAG-3', R: 5'-CG CTTCTGCCTC-3') and a 446-bp fragment was amplified from exon 3 (GenBank NM AF095593, F: 5'-CTGTGCTCA TGTTGTGTCAC-3', R: 5'-CCTGGTGCCAATTTCAAG TTC-3') (8). Polymerase chain reaction-single strand conformation polymorphism (PCR-SSCP) were carried out on the PCR products without non-special amplification (16). Purified PCR samples were also sequenced with an ABI PRISM 310 genetic analyzer (PE Biosystems) to screen for novel mutations.

Statistical analysis. All statistical analyses were performed by the SPSS 13.0 software package for Windows. The $\chi^{2}$ test and Fisher's exact test (2-sided) were used to analyze the correlations between Cav-1 expression and the patients' clinico- pathological parameters. P-values of $<0.05$ were considered to be statistically significant.

\section{Results}

Expression of Cav-1 protein. The red signal was clearly observed under fluorescence microscope at the sites labeled with QDs, showing the existence of Cav-1 protein, indirectly after the slides were incubated with anti-Cav-1 primary antibody, biotinylated goat anti-rabbit IgG and streptavidin conjugated quantum dots (605 $\mathrm{nm}$ QDs).

Cav-1 was highly expressed in cytoplasm and cytomembrane in type I pneumocytes, capillary endothelial cells, fibroblasts and smooth muscle cells (Figs. 1A and 2C). The normal bronchial epithelium, including Clara cells (Fig. 2D), and type II pneumocytes (Fig. 2C), demonstrated negative staining for Cav-1. Cav-1 staining was generally observed in some tumor cells of NSCLC specimens detected by the two different excitation wavelengths (blue and ultraviolet light) (Fig. 2A, B). Positive staining of Cav-1 was observed only in 

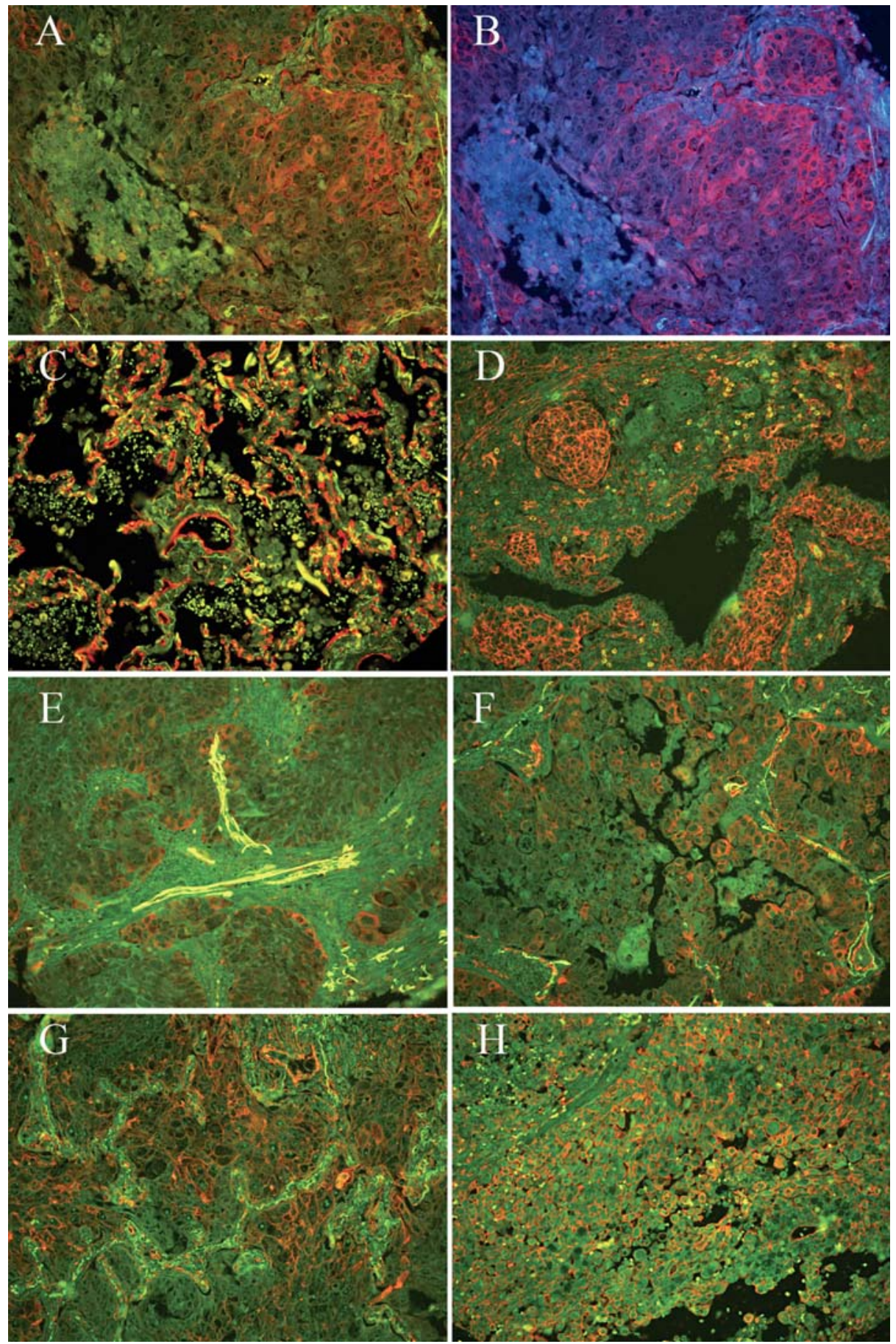

Figure 2. Immunofluorescence histochemical staining for Cav-1 in normal lung tissues and NSCLCs. Positive staining of Cav-1 is clearly observed upon different wavelength excitations [(A), blue light; (B), ultraviolet light]. (C) Strong staining of Cav-1 in the capillary endothelium cells, type 1 pneumocytes and fibroblasts, but negative staining in the cast-off epithelium cell (white arrow). (D) Positive staining of Cav-1 in the SCCs, especially on the cell membranes, but negative staining in the bronchial epithelium (white arrow). (E) Positive staining of Cav-1 in the peripheral cells in carcinomatous nests. (F) Positive staining of Cav-1 in AC tumor cells. (G) Strong Cav-1 staining in squamous cancer cells, not in the adenocarcinoma cells of ASC (white arrow). (H) Strong staining of Cav-1 in LCLC tumor cells. Magnification x200.

the peripheral cancer nests of some SCCs (Fig. 2E). No bronchioloalveolar carcinomas proved to be Cav-1-positive (Fig. 1D). Only 1 of 7 papillary adenocarcinoma was Cav-1 positive. However, there was Cav-1-positive staining of stroma in the AC and SCC samples (Fig. 1E and F). Immunofluorescence staining for Cav-1 was positive in all of the control group (20/20), whereas $49.3 \%(69 / 140)$ of the NSCLCs expressed Cav-1 $\left(\chi^{2}=18.234, \mathrm{P}=0.000\right)$.
Table I shows the relationship between Cav-1 expression and different clinicopathologic parameters in univariate analyses. ACs (16.7\%, Fig. 1C, F), ACCs (38.4\%, Fig. 2G), SCCs $(67.1 \%$, Figs. 1B and 2D) and LCLCs $(66.7 \%$, Fig. 2H) displayed Cav-1-positive staining, suggesting a gradient of Cav-1 expression according to tumor histotype-related aggressiveness. Significant difference of Cav-1 expression was observed between SCCs and ACs $(\mathrm{P}=0.000)$. Statistical 
Table II. Associations between expression of Cav-1 protein and clinicopathological features in patients with lung squamous cell carcinomas.

\begin{tabular}{|c|c|c|c|c|c|}
\hline \multirow[b]{2}{*}{ Variables } & \multicolumn{3}{|c|}{ Cav-1 } & \multirow[b]{2}{*}{$\chi^{2}$ value } & \multirow[b]{2}{*}{ P-value } \\
\hline & Negative & Positive & Positive rate $(\%)$ & & \\
\hline Grade & & & & 0.579 & 0.447 \\
\hline G1-2 & 8 & 21 & 72.4 & & \\
\hline G3 & 19 & 34 & 64.2 & & \\
\hline TNM stage & & & & 2.960 & 0.085 \\
\hline I & 23 & 37 & 61.7 & & \\
\hline $\mathrm{II}+\mathrm{III}$ & 4 & 18 & 81.8 & & \\
\hline $\mathrm{N}$ classification & & & & 4.442 & 0.035 \\
\hline $\mathrm{N}_{0}$ & 24 & 37 & 62.3 & & \\
\hline $\mathrm{N}_{1-3}$ & 3 & 18 & 85.7 & & \\
\hline
\end{tabular}

Table III. Associations between expression of Cav-1 protein and clinicopathological features in patients with lung adenocarcinomas.

\begin{tabular}{|c|c|c|c|c|c|}
\hline \multirow[b]{2}{*}{ Variables } & \multicolumn{3}{|c|}{ Cav-1 } & \multirow[b]{2}{*}{$\chi^{2}$ value } & \multirow[b]{2}{*}{$\mathrm{P}$-value } \\
\hline & Negative & Positive & Positive rate $(\%)$ & & \\
\hline Grade $^{a}$ & & & & 0.003 & 1.000 \\
\hline G1-2 & 19 & 5 & 20.8 & & \\
\hline G3 & 8 & 2 & 20.0 & & \\
\hline TNM stage & & & & 0.105 & 1.000 \\
\hline I & 27 & 5 & 15.6 & & \\
\hline II+III & 8 & 2 & 20.0 & & \\
\hline $\mathrm{N}$ classification & & & & 0.105 & 1.000 \\
\hline $\mathrm{N}_{0}$ & 27 & 5 & 15.6 & & \\
\hline $\mathrm{N}_{1-3}$ & 8 & 2 & 20.0 & & \\
\hline
\end{tabular}

a Bronchiolo-alveolar carcinoma (BAC) was not graded.

correlation was found between the enhanced Cav-1 expression and the advanced stage (stage I vs. stage II+III, $\mathrm{P}=0.045$ ). In addition, high-expression of Cav-1 was associated with the $\mathrm{N}$ classification. In contrast, there was no correlation between the positivity of Cav-1 expression and age, or gender.

Positive correlation was observed between high-expression of Cav-1 protein and lymph node metastasis of SCC. No significant association was found between staining intensity of Cav-1 and the other clinicopathological parameters of SCC (Table II). No correlation was detected between Cav-1 expression and clinicopathological parameters of AC (Table III).

Mutation of Cav-1 in the NSCLCs. To determine whether Cav-1 expression was associated with gene mutation on exons 1 and 3, we performed PCR-SSCP and DNA sequencing on 30 tumor specimen with Cav-1-negative expression and adjacent lung specimens. In all specimens 159 bp on exon 1 and 446 bp on exon 3 were both successfully amplified. Every sequence was verified several times by independent sequencing. No mutations on exon 1 and exon 3 were found in adjacent non-cancerous lung tissues and NSCLC specimens (Fig. 3).

\section{Discussion}

QDs are fluorescent semiconductor nanocrystals with diameters of the order of 2-10 nm or roughly 200-10000 atoms. They are nearly spherical nanocrystals composed of elements from periodic groups of II-VI (CdSe) or III-V (InP). Recently, QDs have been conjugated with many biological molecules, such as proteins, antibodies, and oligonucleotides, as well as streptavidin. So, QDs can effectively and specifically target different biomakers at cellular, tissue, tumor and 


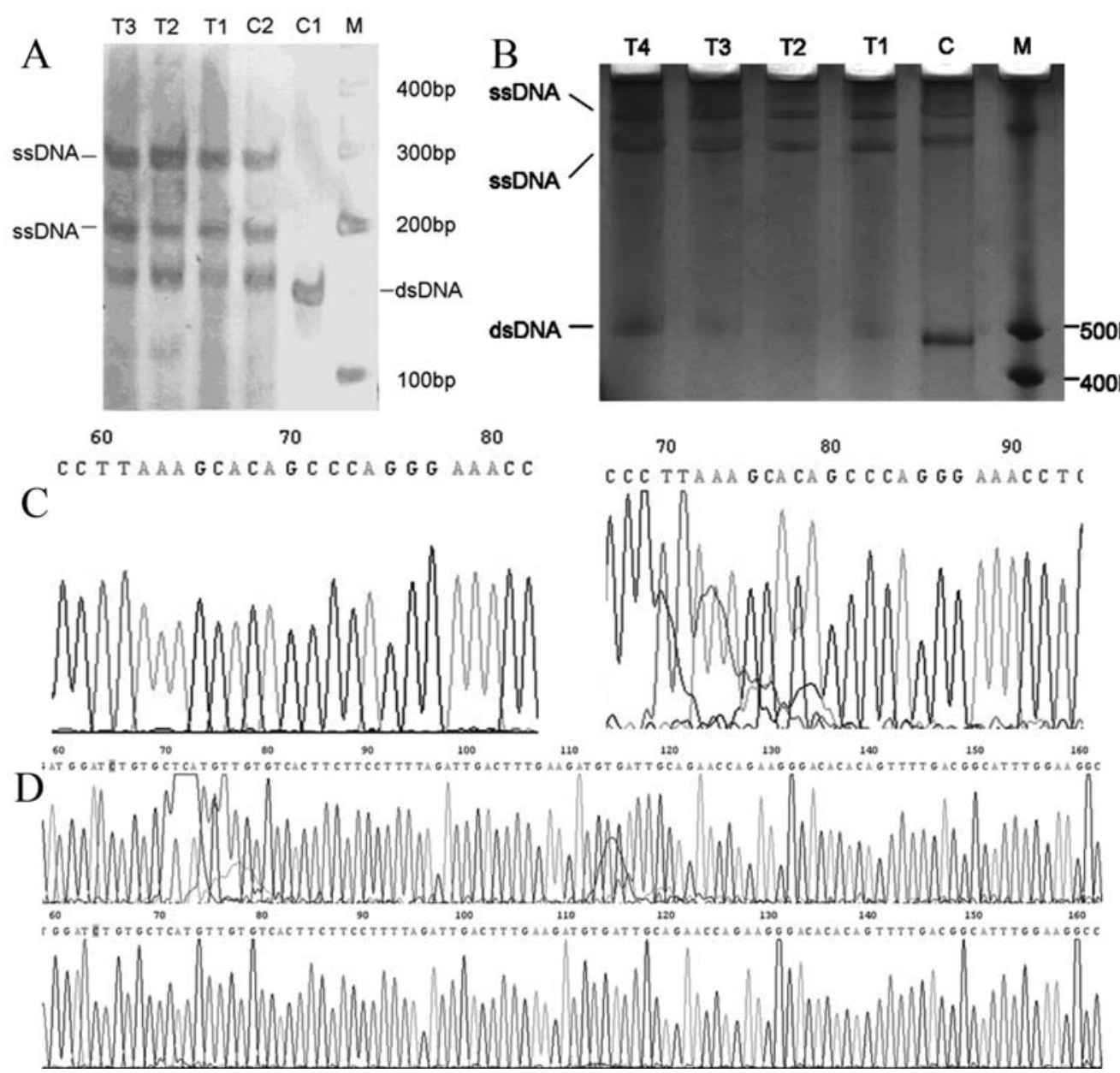

Figure 3. Mutation analysis of Cav-1 exon 1 and exon 3. (A) Cav-1 exon 1 by SSCP analysis. (B) Cav-1 exon 3 by SSCP analysis. The PCR amplification product for exon 1 was 159 bp, exon 3 was 446 bp in size. M, 100 bp ladder markers; C, nearby non-cancerous tissues; ssDNA, single-stranded DNA; dsDNA, double-stranded DNA; T, cancer tissues. (C) Cav-1 gene sequencing in the para-cancerous tissues. (D) Cav-1 gene sequencing in the Cav-1 negative NSCLC tissues. Every sequence was verified several times by independent sequencing. No Cav-1 mutations was observed in human NSCLC specimens.

organ levels, and they have been used as a diagnostic tool for cancer in vivo studies $(17,18)$. The unique optical properties of QDs not only overcome the limitations associated with tissue autofluorescence, but also allow accurate determination of low levels of Cav-1 expression, especially in the lung ACs. Our previous study demonstrated that QDs-IHC is superior to IHC enabling high-throughput application in biopsy and formalin-fixed and paraffin-embedded specimens (13).

In this study, we employed $605 \mathrm{~nm}$ QDs conjugated streptavidin for Cav-1 protein detection, the results show that Cav-1 was highly expressed in cytoplasm and cytomembrane in type I pneumocytes, capillary endothelial cells, fibroblasts and smooth muscle cells, but negatively stained in the normal bronchial epithelium, including Clara cells and type II pneumocytes. These results are in accordance with previous reports $(10,19)$, but Kato et al $(15)$ reported that Cav-1 is expressed in normal bronchial epithelium. We found that immunofluorescence staining for Cav-1 was positive in all 20 cases of the control group, whereas $49.3 \%$ (69/140) of the NSCLCs expressed Cav-1, this result suggested Cav-1 plays an important role in the development of NSCLCs.

In the present study, Cav-1 protein expression was not associated with age or gender, but statistical correlation was found between the enhanced Cav-1 expression and the advanced stage (stage I vs. stage II+III), suggesting that the expression of Cav-1 in tumor cells may be related to tumor invasion. It has been speculated that overexpression of Cav-1 render tumor cells biologically aggressive and invade adjacent normal tissues by induction of the expression of other proteins related to the tumor invasion (10). We also found that Cav-1 expression was significantly increased in metastatic compared with non-metastatic lung cancers, demonstrated that Cav-1 may be regarded as a metastasis promoter in the NSCLCs. These results are in accordance with previous reports $(10,20)$. However, Wikman et al (19) detected Cav-1 protein level by IHC on a tissue microarray containing tumors from 161 patients and normal tissues, Cav-1 expression was not unrelated with clinical stage and lymph node metastasis. We postulated that the Cav-1 deregulated in certain stages of lung cancer, Cav-1 re-expression could adapt to the need of tumor cell invasion and metastasis. Ho et al (21) reported that Cav-1 re-expression in the tumor cells is correlated with the drug resistance and the prognosis of advanced NSCLC patients. Re-introduction of Cav-1 expression into less invasive lung carcinoma cells causes increases in cell invasive ability (22). These results may explain our data in some respect, further study is still required. 
In the present study, ACs (16.7\%), ACCs (38.4\%), SCCs $(67.1 \%)$ and LCLCs $(66.7 \%)$ displayed Cav-1 positive staining, suggesting a gradient of Cav-1 expression according to the histotype-related tumor aggressiveness. Moreover, we observed significant difference of Cav-1 expression between SCCs and ACs, this suggested that the function of Cav-1 may be tissue-dependent (20). Because Cav-1 expression patterns in ACs were admittedly different from those in SCCs, we thought that ACs should be distinguished from SCCs. We found that positive correlation was observed between highexpression of Cav-1 protein and lymph node metastasis of SCCs, suggesting that the expression of Cav-1 in SCCs plays a critical role in modification of biological activity of tumor cells metastasis. We recently reported that Cav-1 expression level is correlated positively with clinical TNM stage and histologic grade of tongue SCC (23). Other studies have reported that overexpression of Cav-1 in esophageal SCC is correlated with $\mathrm{T}$ factor, lymphatic invasion, vein invasion, differentiation and overall survival of patients (24). Based on the results of the esophageal carcinomas and our present results, it suggested that Cav-1 expressed in the same histologic type of carcinoma from different organs might have similar function. In this study, no significant association was found between Cav-1 staining intensity and TNM stage, $\mathrm{T}$ classification, or histopathological grading. On the contrary, Kato et al (15) reported that expression of Cav-1 was significantly correlated with $\mathrm{T}$ classification. However, no correlation was detected between Cav-1 expression and clinicopathologic parameters of ACs in this study. These results are not in accordance with previous reports (18), due to the relatively small number of patients.

$\mathrm{CpG}$ island methylation and gene mutation are two possible ways leading to the loss of Cav-1 expression. A previous study reported that loss of Cav-1 protein expression was not correlated to the methylation status in the promoter region of Cav-1 gene (19). However, the presence of Cav-1 gene mutation was controversial. Cav-1 gene has three exons. Exon 1 and exon 3 mutations were often detected, because the GC content of exon 2 is as high as $62.9 \%$ that by PCR method it is difficult to amplify. Hayashi et al (16) reported a mutation in Cav-1 at codon 132 (P132L) in $16 \%$ of cases in 92 primary human breast cancers, the mutationpositive cases were mostly invasive scirrhous carcinomas. In this study, we showed that no mutation was detected in exon 1 and exon 3 in 30 cases of lung cancer tissues and noncancerous lung tissues. The absence of methylation and somatic mutations in Cav-1 further supports up-regulation of Cav-1 in a proportion of NSCLCs (19). Our findings suggest that more mutation sites may exist requiring further research to confirm. Li et al (25) identified six novel Cav-1 mutations associated with ER $\alpha$-positive breast cancers (W128Stop, Y118H, S136R, I141T, Y148H and Y148S).

In conclusion, the present study suggests that Cav-1 immunoreactivity in lung cancer is histotype-dependent, increased Cav-1 expression indicates the malignant progression and high invasion features of NSCLC. Deregulation of Cav-1 expression in the Chinese NSCLC may not be correlated with gene mutation. Therefore, other mechanisms must exist for the invasion-promoting capability of Cav-1 in lung carcinoma.

\section{Acknowledgements}

This research was supported by grants from the National Natural Science Foundation of China (Nos. 30500226; 30900652).

\section{References}

1. Yang L, Parkin DM, Ferlay J, Li L and Chen Y: Estimates of cancer incidence in China for 2000 and projections for 2005. Cancer Epidemiol Biomarkers Prev 14: 243-250, 2005.

2. Galbiati F, Volonte D, Brown AM, et al: Caveolin-1 expression inhibits Wnt/beta-catenin/Lef-1 signaling by recruiting betacatenin to caveolae membrane domains. J Biol Chem 275: 23368-23377, 2000

3. Cerezo A, Guadamillas MC, Goetz JG, et al: The absence of caveolin-1 increases proliferation and anchorage-independent growth by a Rac-dependent, Erk-independent mechanism. Mol Cell Biol 29: 5046-5059, 2009.

4. Bartholomew JN, Volonte D and Galbiati F: Caveolin-1 regulates the antagonistic pleiotropic properties of cellular senescence through a novel Mdm2/p53-mediated pathway. Cancer Res 69: 2878-2886, 2009.

5. Wiechen K, Diatchenko L, Agoulnik A, et al: Caveolin-1 is down-regulated in human ovarian carcinoma and acts as a candidate tumor suppressor gene. Am J Pathol 159: 1635-1643, 2001.

6. Bender FC, Reymond MA, Bron C and Quest AF: Caveolin-1 levels are down-regulated in human colon tumors, and ectopic expression of caveolin- 1 in colon carcinoma cell lines reduces cell tumorigenicity. Cancer Res 60: 5870-5878, 2000.

7. Zhang H, Su L, Müller S, et al: Restoration of caveolin-1 expression suppresses growth and metastasis of head and neck squamous cell carcinoma. Br J Cancer 99: 1684-1694, 2008.

8. Hurlstone AF, Reid G, Reeves JR, et al: Analysis of the CAVEOLIN-1 gene at human chromosome $7 q 31.1$ in primary tumours and tumour-derived cell lines. Oncogene 18: 1881-1890, 1999.

9. Karam JA, Lotan Y, Roehrborn CG, Ashfaq R, Karakiewicz PI and Shariat SF: Caveolin-1 overexpression is associated with aggressive prostate cancer recurrence. Prostate 67: 614-622, 2007.

10. Yoo SH, Park YS, Kim HR, et al: Expression of caveolin-1 is associated with poor prognosis of patients with squamous cell carcinoma of the lung. Lung Cancer 42: 195-202, 2003.

11. Zhang ZB, Cai L, Zheng SG, Xiong Y and Dong JH: Overexpression of caveolin-1 in hepatocellular carcinoma with metastasis and worse prognosis: correlation with vascular endothelial growth factor, microvessel density and unpaired artery. Pathol Oncol Res 15: 495-502, 2009.

12. Tholouli E, Sweeney E, Barrow E, Clay V, Hoyland JA and Byers RJ: Quantum dots light up pathology. J Pathol 216: 275-285, 2008.

13. Chen H, Xue J, Zhang Y, Gao J and Yu B: Comparison of quantum dots immunofluorescence histochemistry and conventional immunohistochemistry for the detection of caveolin-1 and PCNA in the lung cancer tissue microarray. J Mol Histol 40: 261-268, 2009.

14. Sauter G, Simon R and Hillan K: Tissue microarrays in drug discovery. Nat Rev Drug Discov 2: 962-972, 2003.

15. Kato T, Miyamoto M, Kato K, et al: Difference of caveolin-1 expression pattern in human lung neoplastic tissue. Atypical adenomatous hyperplasia, adenocarcinoma and squamous cell carcinoma. Cancer Lett 214: 121-128, 2004.

16. Hayashi K, Matsuda S, Machida K, et al: Invasion activating caveolin-1 mutation in human scirrhous breast cancers. Cancer Res 61: 2361-2364, 2001.

17. Gao XY, Cui RM, Levenson L, Chung WK and Nie S: In vivo cancer targeting and imaging with semiconductor quantum dots. Nat Biotechnol 22: 969-976, 2004.

18. Yu X, Chen L, Deng Y, et al: Fluorescence analysis with quantum dot probes for hepatoma under one- and two-photon excitation. J Fluoresc 17: 243-247, 2007.

19. Wikman H, Seppänen JK, Sarhadi VK, et al: caveolin-1 as tumor markers in lung cancer detected by combined use of cDNA and tissue microarrays. J Pathol 203: 584-593, 2004. 
20. Cassoni P, Daniele L, Maldi E, et al: Caveolin-1 expression in lung carcinoma varies according to tumour histotype and is acquired de novo in brain metastases. Histopathology 55: 20-27, 2009.

21. Ho CC, Kuo SH, Huang PH, Huang HY, Yang CH and Yang PC: Caveolin-1 expression is significantly associated with drug resistance and poor prognosis in advanced non-small cell lung cancer patients treated with gemcitabine-based chemotherapy. Lung Cancer 59: 105-110, 2008.

22. Ho CC, Huang PH, Huang HY, Chen YH, Yang PC and Hsu SM: Up-regulated caveolin-1 accentuates the metastasis capability of lung adenocarcinoma by inducing filopodia formation. Am $\mathbf{J}$ Pathol 161: 1647-1656, 2002.
23. Xue J, Chen H, Diao L, Chen X and Xia D: Expression of caveolin-1 in tongue squamous cell carcinoma by quantum dots. Eur J Hist 54: 99-103, 2010.

24. Ando T, Ishiguro $\mathrm{H}$, Kimura $\mathrm{M}$, et al: The overexpression of caveolin-1 and caveolin-2 correlates with a poor prognosis and tumor progression in esophageal squamous cell carcinoma. Oncol Rep 18: 601-609, 2007.

25. Li T, Sotgia F, Vuolo MA, et al: Caveolin-1 mutations in human breast cancer: functional association with estrogen receptor alpha-positive status. Am J Pathol 168: 1998-2013, 2006. 